\title{
The isolated caecal patch lesion: a clinical, endoscopic and histopathological study
}

\author{
Anju Ekanayaka, ${ }^{1}$ John T Anderson, ${ }^{2}$ Michele E Lucarotti, ${ }^{3}$ Roland M Valori, ${ }^{4}$ \\ Neil A Shepherd (1) ${ }^{1}$
}

${ }^{1}$ Gloucestershire Cellular Pathology Laboratory, Cheltenham, UK

${ }^{2}$ Department of Gastroenterology, Cheltenham General Hospital, Cheltenham, UK

${ }^{3}$ Department of Colorectal Surgery, Gloucestershire Royal Hospital, Gloucester, UK

${ }^{4}$ Department of Gastroenterology,

Gloucestershire Royal Hospital, Gloucester, UK

\section{Correspondence to} Professor Neil A Shepherd, Gloucestershire Cellular Pathology Laboratory, Cheltenham General Hospital, Sandford Road, Cheltenham, Gloucester GL53 7AN, UK; neil. a.shepherd@nhs.net

Received 5 August 2019 Revised 28 August 2019 Accepted 29 August 2019 Published Online First 25 September 2019
Check for updates

(C) Author(s) (or their employer(s)) 2020. No commercial re-use. See rights and permissions. Published by BMJ.

To cite: Ekanayaka A, Anderson JT, Lucarotti ME, et al. J Clin Pathol

2020;73:121-125.

\begin{abstract}
Objective To describe and investigate the potential causes of the isolated caecal patch lesion, a previously undescribed endoscopic phenomenon of a lesion fulfilling endoscopic and histopathological criteria for chronic inflammatory bowel disease but without evidence of similar inflammatory pathology elsewhere at colonoscopy.
\end{abstract}

Methods Cases were collected prospectively by one specialist gastrointestinal pathologist over a 10-year period. Full endoscopic and histopathological analysis was undertaken and follow-up sought to understand the likely cause(s) of the lesions.

Results Six cases are described. Two had very close links with ulcerative colitis, one predating the onset of classical distal disease and the other occurring after previous demonstration of classical distal ulcerative colitis. Two occurred in younger patients and we postulate that these lesions may predict the subsequent onset of chronic inflammatory bowel disease. Finally two can be reasonably attributed to the effects of nonsteroidal inflammatory agent therapy.

Conclusions Caecal patch lesions can be demonstrated in isolation. Despite the strong association of caecal patch lesions with ulcerative colitis, solitary lesions may well have disparate causes but nevertheless possess a close relationship with chronic inflammatory bowel disease.

\section{INTRODUCTION}

Various clinical, endoscopic and pathological features are used to differentiate ulcerative colitis (UC) from its main differential diagnosis, Crohn's disease. Continuity of disease, especially in the colon, is one of the most important, discontinuity of disease being a key feature of intestinal Crohn's disease. $^{12}$ UC is characteristically a continuous disease, involving the rectum and a variable extent of the colon. Despite this, it is now recognised that UC itself can be discontinuous. There may be continuous disease in the rectum and distal colon, a normal proximal colon and involvement of the appendix, a situation that has been termed 'the appendiceal skip lesion of UC'. ${ }^{34}$ We also recognise a close relationship between diverticular disease and UC. 'Diverticular colitis' may represent discontinuous UC afflicting the sigmoid colon and there are now many established cases where diverticular colitis, with the luminal mucosa of the sigmoid colon showing characteristic histopathological features of chronic inflammatory bowel disease (CIBD), but with an entirely normal rectal mucosal pathology, has preceded the subsequent onset of classical UC with involvement of the rectal mucosa by chronic active inflammation and the distinctive features of UC. ${ }^{5} 6$

The link to discontinuous colonic involvement in chronic UC is now recognised with both appendiceal involvement and the 'caecal patch lesion'. ${ }^{7-11}$ Similar to the appendiceal skip lesion of UC, there is continuous distal disease of UC, with more proximal colonic normality and then an isolated focus of caecal involvement by chronic active inflammatory disease, producing the caecal patch lesion. This has identical histological appearances to those seen in the more distal colon and rectum with the characteristic changes of active CIBD. ${ }^{711} 12$

The caecal patch lesion was first defined in 1958 by Lumb and Protheroe, who described eight cases in which the features of UC occurred as 'an island' within normal caecal mucosa, with a tendency to occur in the lower caecum opposite the ileocaecal valve. ${ }^{12}$ Since that initial description, there have been several other reports, case series, reviews and studies published to elucidate the prevalence and significance of caecal patch lesions in UC. ${ }^{7-9} 11$ 13-16 Caecal patch lesions indicate the likelihood of more severe distal disease although the lesion itself is associated with a better response to therapy. ${ }^{13}$ They are more common in younger male patients with UC and associated with more pronounced symptomatology, especially abdominal pain, rectal bleeding and diarrhoea. ${ }^{11} 14$ In contrast, the presence of appendiceal orifice inflammation does not predict the prognosis of UC, including remission rate, relapse rate, proximal disease extension and the need for proctocolectomy. ${ }^{15} 16$

This paper describes our experience with the 'isolated caecal patch lesion' (ICPL), where the caecal lesion, as defined, is the only significant finding at colonoscopy. To our knowledge, this phenomenon has not been recognised in the literature. Because of this, it is appropriate that this phenomenon is rigorously defined. By the ICPL, we infer a variable clinical presentation and an endoscopic appearance of an isolated and localised area of inflamed mucosa contained within the caecum, with histopathological features in keeping with CIBD. The latter includes diffuse chronic inflammation of the lamina propria, active inflammation, whether intraepithelial polymorphs and/or established crypt abscesses, evidence of crypt architectural distortion, mucin depletion, however variable, and no evidence of granulomatous pathology. Those histopathological features are, of course, suggestive 


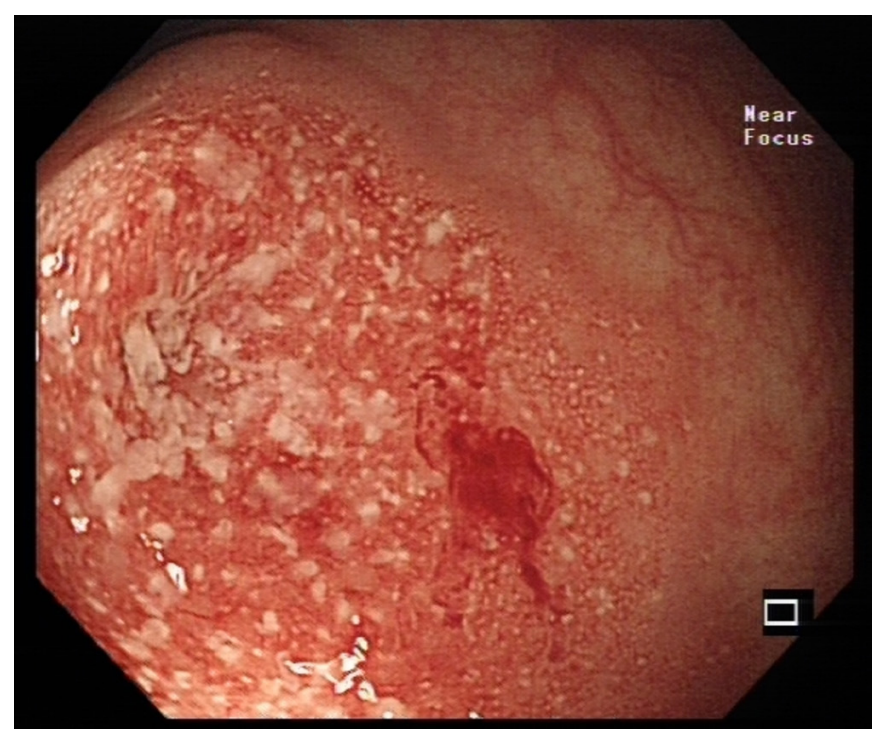

Figure 1 The isolated caecal patch lesion at endoscopy in patient 1. There is a discrete oval-shaped area with diffuse hyperaemia and white punctate patches, some coalescent, within.

of UC but, as part of the definition, we imply that the remaining colonoscopy shows no other features to suggest CIBD elsewhere (any incidental pathology would still be acceptable) and knowledge that biopsies taken elsewhere in the colon and rectum, especially the rectum, show no evidence of CIBD. Further, in none of our cases was there any evidence, at the time of the relevant colonoscopy, whether clinical, radiological, endoscopic or histological, to suggest CIBD elsewhere in the gastrointestinal tract, especially, of course, in the terminal ileum and rectum.

\section{MATERIALS AND METHODS}

The six cases described here represent a retrospective series from the files of one specialist gastrointestinal pathologist (NAS). All patients derive from our two hospitals in Gloucestershire and were collected during a 10-year period from 2006 to 2016. The patients included in this study had a diagnosis of ICPL, demonstrated endoscopically and confirmed histologically as previously defined. Endoscopically all patients had a patch of disease typified by erythema, loss of vascular pattern and granularity of the mucosa identical to that usually seen in UC in the distal colon and rectum. The lesion was usually symmetrical around the appendix orifice extending $1-5 \mathrm{~cm}$ distally with a relatively sharp cut-off to normal-appearing mucosa. In none of our patients did the lesion extend beyond the caecal pole. The remaining colon and rectum were either normal or harboured incidental pathology not relevant to CIBD. Biopsies taken from the caecal patch lesion showed the pathological features of active
CIBD and all other colonoscopic biopsies showed no histological evidence of CIBD.

Baseline data, including gender, age at diagnosis, initial symptoms/indication and endoscopic details were extracted by reviewing the medical records of the patients. These medical records were reviewed for additional relevant details including drug history, any treatment of the presenting symptoms, the response to that treatment, subsequent symptoms and follow-up endoscopy. After data collection, identifying medical record numbers and names were removed, and unique identifiers were created to ensure patient confidentiality.

All histopathological materials, from the intestines of all patients, were reviewed. The inflammatory changes present in the caecal biopsies were scored according to the activity index developed by Geboes and his colleagues. ${ }^{17}$

\section{RESULTS}

The clinical and endoscopic details are presented in table 1 . The patients' ages ranged from 28 to 57, three women and three men. Four patients presented with chronic diarrhoea; in three of them, there was also per-rectal (PR) bleeding. One patient had had one episode of fresh PR bleeding only. One presented with chronic abdominal pain. One patient had a strong family history of UC: the mother and grandmother had had UC. Two patients had a history of non-steroidal inflammatory agents' (NSAIDs) usage.

\section{Endoscopic findings}

All patients had isolated inflammatory changes confined to the caecum. The lesions were easily distinguishable from the surrounding mucosa. Optical evaluation revealed a discrete spherical or oval-shaped area with diffuse hyperaemia (figure 1). Individual cases exhibited varying degrees of endoscopically visible erythema. Within this background change, there were discrete white punctate patches which may or may not have coalesced. The involved area was friable and demonstrated either contact bleeding, with minimal trauma, or profound oozing after biopsy. The endoscopic features had the appearance of superficial erosive or microulcerative change. The mucosa was minimally nodular in appearance and not raised compared with the surrounding mucosa. Although the lesions were well localised, the margins were not well circumscribed. The erythematous areas blended with the adjacent normal mucosa. Narrow band imaging revealed dark areas, reflecting the increased vascularity and inflammation present (figure 2). Incidental findings, at colonoscopy, included uncomplicated diverticular disease in one patient and one small polyp in the transverse colon in another. The remaining colonoscopy was normal in all the other patients.

\section{Histological findings}

The histological features are summarised in table 2. Architectural abnormalities, in the form of crypt architectural changes,

Table 1 Clinical and endoscopic data

\begin{tabular}{|c|c|c|c|c|c|c|}
\hline Patient & 1 & 2 & 3 & 4 & 5 & 6 \\
\hline Age at presentation with ICPL & 47 & 57 & 21 & 28 & 55 & 57 \\
\hline Sex & Male & Female & Female & Male & Female & Male \\
\hline Indication for colonoscopy & Chronic abdominal pain & Chronic diarrhoea & $\begin{array}{l}\text { Chronic diarrhoea and PR } \\
\text { bleeding }\end{array}$ & $\begin{array}{l}\text { Chronic diarrhoea and } \\
\text { PR bleeding }\end{array}$ & $\begin{array}{l}\text { Chronic diarrhoea and } \\
\text { PR bleeding }\end{array}$ & PR bleeding \\
\hline Family history of IBD & None & None & Mother and grandmother & None & None & None \\
\hline Drug history & None relevant & None relevant & None relevant & None relevant & NSAIDs & NSAIDs \\
\hline Additional endoscopic findings & Polyp in TC & None & None & None & None & None \\
\hline
\end{tabular}

IBD, inflammatory bowel disease; ICPL, isolated caecal patch lesion; NSAID, non-steroidal inflammatory agent; PR, per-rectal; TC, transverse colon. 


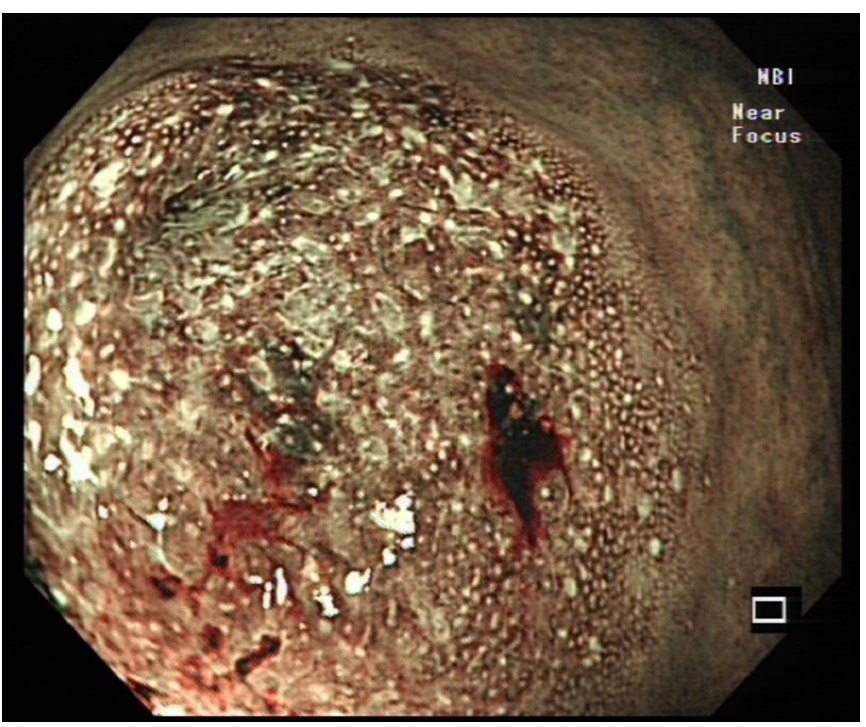

Figure 2 A narrow band image of the isolated caecal patch lesion in figure 1. The dark areas represent the increased vascularity due to the inflammation present.

and acute and chronic inflammation were identified in all biopsies taken from the caecum (figure 3 ). The histological appearances very much reflected the endoscopic appearances. All the cases showed similar features with remarkably similar levels of active inflammation (table 2). All patients showed no evidence of active inflammation/CIBD at any site apart from the caecum at the relevant colonoscopy.

\section{Follow-up findings and associations}

The follow-up details are presented in table 3. Two patients showed very clear associations with CIBD. Patient 1 , with no previous history and only a caecal patch lesion at the index colonoscopy, re-presented 6 months later with classical UC, in a proctitis distribution, when the caecal patch lesion was also present. Patient 2 had previously documented UC, in a 'distal colitis' distribution, 5 years previously. This was treated and the patient was in remission until the diagnosis of isolated caecal patch was made, after she had represented with chronic diarrhoea. At that time, there was only endoscopic evidence of a caecal patch lesion with no evidence of UC elsewhere despite extensive biopsies of the colon and rectum.

Two younger patients, aged 21 and 28 (patients 3 and 4), were effectively treated for CIBD and remain symptomatically free of disease 3 years and 5 years after the diagnoses of ICPLs, respectively. It may be that the presentation with ICPLs is a prelude to the subsequent development of UC but, at the current time, there is no positive evidence of this.

\begin{tabular}{lcccccc}
\hline Table 2 & Grading of histological variables (after Geboes & et $\mathrm{I}^{17}$ ) \\
\hline Patient & $\mathbf{1}$ & $\mathbf{2}$ & $\mathbf{3}$ & $\mathbf{4}$ & $\mathbf{5}$ & 6 \\
\hline Structural (architectural) changes & 1 & 1 & 2 & 2 & 2 & 2 \\
Chronic inflammatory infiltrate & 3 & 2 & 3 & 3 & 3 & 2 \\
Lamina propria eosinophils & 0 & 0 & 0 & 0 & 0 & 0 \\
Lamina propria neutrophils & 3 & 1 & 2 & 2 & 2 & 1 \\
Neutrophils in epithelium & 2 & 0 & 2 & 2 & 1 & 0 \\
Crypt destruction & 1 & 0 & 2 & 0 & 0 & 0 \\
Erosion or ulceration & 0 & 0 & 0 & 0 & 0 & 0 \\
\hline
\end{tabular}

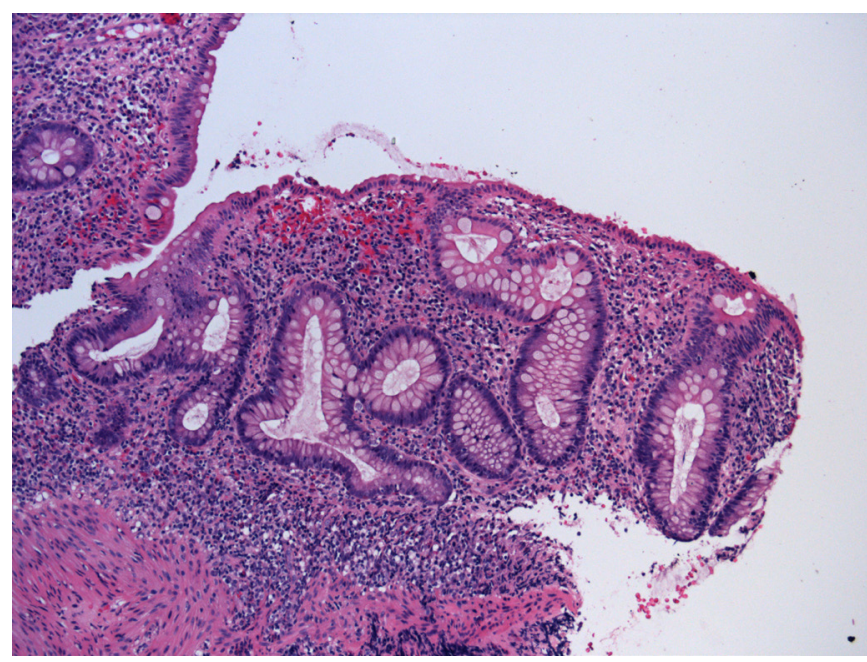

Figure 3 A biopsy from the isolated caecal patch lesion in the caecum of patient 2. At this power, the crypt architectural distortion and diffuse chronic inflammation are obvious. There was more patchy active inflammation. The features are very similar to those seen in ulcerative colitis.

Finally, two patients had been on NSAID therapy. Patients 5 and 6 had both been taking diclofenac (voltarol) for 2 years because of osteoarthritis. There was no significant drug history for the other four patients. NSAIDs are a well-recognised cause of inflammatory pathology in the intestines and a cause of mimicry of CIBD. In both patients, the NSAIDs were stopped with no clinical or endoscopic evidence of disease in the intestines subsequently. We believe the NSAIDs are the likely cause of the caecal patch lesions in these two patients.

\section{DISCUSSION}

This study has demonstrated that classical caecal patch lesions can be seen in isolation at colonoscopy. Here we describe six such cases and provide evidence that they represent a disparate group, in terms of causation. Two cases show features demonstrating a strong relationship with UC. One patient presented with an ICPL but subsequently re-presented with classical UC in a 'proctitis' distribution. This is similar to the now well-documented occurrence of patients with isolated diverticular colitis re-presenting subsequently with classical UC, despite there being no evidence of the latter diagnosis at the index endoscopy. ${ }^{56}$ Perhaps it is no surprise that, in one case, ICPL occurred in a patient already known to have had UC previously. We recognise that UC can return to effective normality, both endoscopically ${ }^{18} 19$ and histopathologically ${ }^{20}$ but this study is the first to identify that recrudescent disease can be solely with an ICPL.

Two cases have occurred in younger patients and we postulate that these patients may eventually re-present with classical UC, the ICPLs perhaps being the harbinger of more classical UC disease although some may regard that as speculative. Those patients had no other significant history and, in particular, no relevant drug history.

NSAIDs are implicated in a wide range of gastrointestinal pathology. $^{21}$ In the upper gastrointestinal tract, they are an important cause of gastritis, duodenitis and peptic ulceration. ${ }^{2122}$ In the small intestine, long-term inflammation can result in stricturing and 'diaphragm disease'. ${ }^{23}$ Diaphragm disease due to NSAIDs has also been seen in the proximal colon and NSAIDs have also been found responsible for inducing acute appendicitis and acute 
Table 3 Patient follow-up details

\begin{tabular}{lllllll}
\hline & Patient 1 & Patient 2 & Patient 3 & Patient 4 & Patient 5 & Patient 6 \\
\hline Diagnosis & ICPL $\rightarrow$ UC & UC $\rightarrow$ ICPL & Possible UC & Possible UC & NSAIDs & NSAIDs \\
Follow-up treatment & Mesalamine & Mesalamine & Mesalamine & Mesalamine & None & None \\
Treatment response & Good & Good & Excellent & Excellent & Full remission & Full remission \\
\hline
\end{tabular}

ICPL, caecal patch lesion;NSAID, non-steroidal inflammatory agent; UC, ulcerative colitis.

diverticulitis in patients with sigmoid colonic diverticular disease. ${ }^{21}$ NSAIDs also have a close relationship with CIBD. ${ }^{24} 25$ They are recognised to induce relapses of $\mathrm{UC}^{2425}$ and some have postulated that they may even cause CIBD. ${ }^{26}$ Mimicry of CIBD by NSAID enterocolopathy is well described. ${ }^{27}{ }^{28}$ It is apposite therefore that two of our patients had been on long-term diclofenac, an NSAID agent well recognised to cause gastrointestinal pathology. ${ }^{29}$ The fact that both patients are in clinical remission from gastrointestinal symptoms after discontinuing diclofenac implies that NSAIDs may well be the cause of the ICPL in these patients although it is impossible to exclude other potential aetiologies. Indeed, even in these patients, the ICPLs may be a harbinger for the development of subsequent CIBD.

The pathogenesis of ICPLs, when they occur in association with classical UC, remains uncertain. What is notable is that these lesions occur at sites of relative faecal stasis. The same argument can be applied to the appendiceal 'skip lesion of UC' whereby active inflammation is notably confined to the mucosa of the appendix, in the absence of active inflammation elsewhere in the proximal large intestine, but with classical UC more distally. Indeed the link with faecal stasis can also be implied to explain the association of diverticular colitis with UC, especially those cases that present initially with diverticular colitis and then go on to present with classical UC. ${ }^{56}$ In those cases, the initial inflammatory pathology occurs at sites of considerable faecal stasis, sigmoid colonic diverticula. Furthermore, we recognise that the most common site for UC, especially at the index presentation, is the rectum, another site that is clearly associated with faecal stasis. Could faecal stasis, then, be an important pathogenetic factor in the development of these 'skip lesions' of UC and, ultimately, in the causation of UC itself? There has been a considerable body of work researching the relationship between faecal stasis and mucolysis and there has also been much research on the influence of mucin pathology in the genesis of UC. ${ }^{30-32}$ Do these cases, wherein isolated disease occurs at sites of faecal stasis, provide support for these theories about the aetiopathogenesis of chronic UC?

This study has introduced a novel endoscopic and pathological phenomenon, the ICPL, and has provided evidence to suggest potential causes in the six cases. There is no doubt that the lesion has a close relationship with chronic UC. In none of our cases was there any clinical, endoscopic or, especially, pathological evidence of Crohn's disease but Crohn's disease remains a possible, if unlikely, cause of the endoscopic finding of ICPL. We

\section{Take home messages}

- Caecal patch lesions are an increasingly recognised feature of chronic ulcerative colitis.

- Caecal patch lesions show characteristic endoscopic and histological features.

- Caecal patch lesions may predate the onset of classical distal ulcerative colitis.

- Caecal patch lesions may be caused by diseases other than ulcerative colitis. postulate that NSAIDs are an occasional cause of these lesions. The ICPL highlights the interesting occurrence of UC at sites of faecal stasis and hints at a potential pathogenetic factor in the development of chronic UC.

\section{Handling editor Dhirendra Govender}

Contributors NAS collected the initial data. AE collected and organised the data on the pathological findings and AE and NAS assessed the pathology equally and contributed to the writing and editing of the manuscript equally. JTA, MEL and RMV all contributed equally to the collection and organisation of clinical and endoscopic data and the writing and editing of the manuscript. JTA provided the endoscopic photographs.

Funding The authors have not declared a specific grant for this research from any funding agency in the public, commercial or not-for-profit sectors.

Competing interests None declared.

Patient consent for publication Not required.

Provenance and peer review Not commissioned; externally peer reviewed.

Data availability statement Data are available on reasonable request.

ORCID iD

Neil A Shepherd http://orcid.org/0000-0003-4228-6580

\section{REFERENCES}

1 Feakins RM. Inflammatory bowel disease biopsies: updated British Society of gastroenterology reporting guidelines. J Clin Pathol 2013;66:1005-26.

2 Langner C, Magro F, Driessen A, et al. The histopathological approach to inflammatory bowel disease: a practice guide. Virchows Arch 2014;4(5 Suppl 1):511-27.

3 Davison AM, Dixon MF. The appendix as a 'skip lesion' in ulcerative colitis. Histopathology 1990;16:93-5.

4 Park SH, Loftus EV, Yang S-K. Appendiceal SKIP inflammation and ulcerative colitis. Dig Dis Sci 2014:59:2050-7.

5 Shepherd NA. Diverticular disease and chronic idiopathic inflammatory bowel disease: associations and masquerades. Gut 1996:38:801-2.

6 Ludeman L, Shepherd NA. What is diverticular colitis? Pathology 2002;34:568-72.

7 D'Haens G, Geboes K, Peeters M, et al. Patchy cecal inflammation associated with distal ulcerative colitis: a prospective endoscopic study. Am J Gastroenterol 1997;92:1275-9.

8 Ladefoged K, Munck LK, Jorgensen F, et al. Skip inflammation of the appendiceal orifice: a prospective endoscopic study. Scand J Gastroenterol 2005:40:1192-6.

9 Katz J, Shepela C. Clinical significance of isolated peri-appendiceal lesions in patients with left sided ulcerative colitis. Gastroenterol Res 2011;4:58-63.

10 Feakins RM, Shepherd NA. An update on the pathology of chronic inflammatory bowel disease. In: Pignatelli M, Gallagher P, eds. Recent advances in histopathology. 23rd edn. JP Medical Publishers, 2014: 117-34.

11 Nevin D, Genta R, Saboorian H. The cecal patch associated with a greater prevalence of symptoms in patients with ulcerative colitis. Am J Gastroenterology 2012; 107:S654.

12 Lumb G, Protheroe RH. Ulcerative colitis; a pathologic study of 152 surgical specimens. Gastroenterology 1958;34:381-407.

13 Matsumoto T, Nakamura S, Shimizu M, et al. Significance of appendiceal involvement in patients with ulcerative colitis. Gastrointest Endosc 2002;55:180-5.

14 Yamagishi N, lizuka B, Nakamura T, et al. Clinical and colonoscopic investigation of skipped periappendiceal lesions in ulcerative colitis. Scand J Gastroenterol 2002;37:177-82

15 Byeon J-S, Yang S-K, Myung S-J, et al. Clinical course of distal ulcerative colitis in relation to appendiceal orifice inflammation status. Inflamm Bowel Dis 2005:11:366-71.

16 Park SH, Yang S-K, Park S-K, et al. Atypical distribution of inflammation in newly diagnosed ulcerative colitis is not rare. Can J Gastroenterol Hepatol 2014;28:125-30.

17 Geboes K, Riddell R, Ost A, et al. A reproducible grading scale for histological assessment of inflammation in ulcerative colitis. Gut 2000;47:404-9.

18 Neurath MF, Travis SPL. Mucosal healing in inflammatory bowel diseases: a systematic review. Gut 2012;61:1619-35. 
19 Bryant RV, Burger DC, Delo J, et al. Beyond endoscopic mucosal healing in UC: histological remission better predicts corticosteroid use and hospitalisation over 6 years of follow-up. Gut 2016;65:408-14.

20 Levine TS, Tzardi M, Mitchell S, et al. Diagnostic difficulty arising from rectal recovery in ulcerative colitis. J Clin Pathol 1996;49:319-23.

21 Parfitt JR, Driman DK. Pathological effects of drugs on the gastrointestinal tract: a review. Hum Pathol 2007;38:527-36.

22 Pusztaszeri MP, Genta RM, Cryer BL. Drug-Induced injury in the gastrointestinal tract: clinical and pathologic considerations. Nat Clin Pract Gastroenterol Hepatol 2007:4:442-53.

23 Lang J, Price AB, Levi AJ, et al. Diaphragm disease: pathology of disease of the small intestine induced by non-steroidal anti-inflammatory drugs. J Clin Pathol 1988:41:516-26.

24 Felder JB, Korelitz BI, Rajapakse R, et al. Effects of nonsteroidal antiinflammatory drugs on inflammatory bowel disease: a case-control study. Am J Gastroenterol 2000;95:1949-54.

25 Singh S, Graff LA, Bernstein CN. Do NSAIDs, antibiotics, infections, or stress trigger flares in IBD? Am J Gastroenterol 2009;104:1298-313.
26 Gleeson MH, Davis AJM. Non-Steroidal anti-inflammatory drugs, aspirin and newly diagnosed colitis: a case-control study. Aliment Pharmacol Ther 2003;17:817-25.

27 Shepherd NA. Pathological mimics of chronic inflammatory bowel disease. J Clin Pathol 1991;44:726-33.

28 Stolte M, Hartmann F. Misinterpretation of NSAID-induced Colopathy as Crohn's Disease. Z Gastroenterol 2010;48:472-5.

29 Lee FD. Drug-Related pathological lesions of the intestinal tract. Histopathology 1994;25:303-8.

30 Rhodes JM. Colonic mucus and mucosal glycoproteins: the key to colitis and cancer? Gut 1989;30:1660-6.

31 Pullan RD, Thomas GA, Rhodes M, et al. Thickness of adherent mucus gel on colonic mucosa in humans and its relevance to colitis. Gut 1994;35:353-9.

32 Png CW, Lindén SK, Gilshenan KS, et al. Mucolytic bacteria with increased prevalence in IBD mucosa augment in vitro utilization of mucin by other bacteria. Am J Gastroenterol 2010;105:2420-8. 